\title{
Ghost particle reduction in 3D particle streak velocimetry
}

\author{
C. Tsalicoglou ${ }^{1 *}$, T. Roesgen ${ }^{1}$ \\ ${ }^{1}$ ETH Zürich, Institute of Fluid Dynamics, Zurich, Switzerland \\ *ctsalico@ethz.ch
}

\begin{abstract}
Ghost particles are ambiguities in the process of the 3D-reconstruction of seeding particles detected in short-exposure imaging for volumetric flow velocimetry. 3D Particle Streak Velocimetry (3D-PSV) relies on long-exposure images, where the pathlines of the seeding particles are imaged as streaks. In this work, we demonstrate the inherent suitability of 3D-PSV for ghost particle rejection by calculating the probability of ghost streak generation in different scenarios and comparing our results to simulations.
\end{abstract}

\section{Introduction}

Volumetric velocimetry techniques for fluid flows, such as 3D Particle Tracking Velocimetry (3D-PTV) and Tomographic Particle Image Velocimetry (Tomo-PIV), use the identified discrete locations of tracer particles in two or more camera views to reconstruct the tracers' positions in 3D space, track them over time and retrieve the flow velocity. The reconstruction of the particles in 3D space can result in so-called "ghost particles", which are reconstructed tracer particles for which it remains uncertain whether they are real or artifacts of the reconstruction process. More camera views, tracking, smoothness criteria, and intensitybased procedures are used to improve the reconstruction quality under increasingly demanding requirements for temporal and spatial resolution (e.g., Schanz et al. (2016), Novara et al. (2019)).

In contrast to 3D-PTV, which relies on the reconstruction of points that represent "frozen" particles, 3D Particle Streak Velocimetry (3D-PSV) (e.g., Rosenstiel and Grigat (2010)) uses long-exposure images, where the signature of each particle is its pathline over the exposure time, allowing for the triangulation of pathline segments, or "streaks". The streaks can be treated as linear segments when only the information about the connectivity of their endpoints is used or as curved segments when considering the shape of the connecting curve. We show here that already the use of the endpoint connectivity alone, employed in the reconstruction of linear streaks, allows for a significant reduction in ghost particle generation, without the need to consider additional time-steps or to perform particle tracking over the exposure time.

\section{Methods and Results}

Maas (1992) described the probability of generating ghost particles from images of randomly distributed particles as a function of the particles per pixel, volume depth, and relative position of the cameras. For $n$ imaged particles, the expected number of ambiguous particles $N_{p}$ in a search window $f$ for an image of size $F$, was estimated as $N_{p}=\left(n^{2}-n\right) f / F$.

The estimates were based on considerations in epipolar geometry, with the necessary condition for ghost particle generation being that, given one particle on an epipolar line, an ambiguity occurs as soon as there is at least one more particle on the same epipolar line.

In streak images, twice the number of points must be considered, as the signature of each of the $n$ particles is a line segment with both its endpoints in the same image, and we assume that all particles remain within the field of view during the exposure time. We consider a stereo setup where the cameras have co-planar image planes and parallel axes, as this setup results in parallel epipolar lines, which simplifies the subsequent considerations (Fig. 1(a)). Two scenarios are examined to calculate the probabilities of ghost streak generation: random displacements of the particles within the images and random displacements 


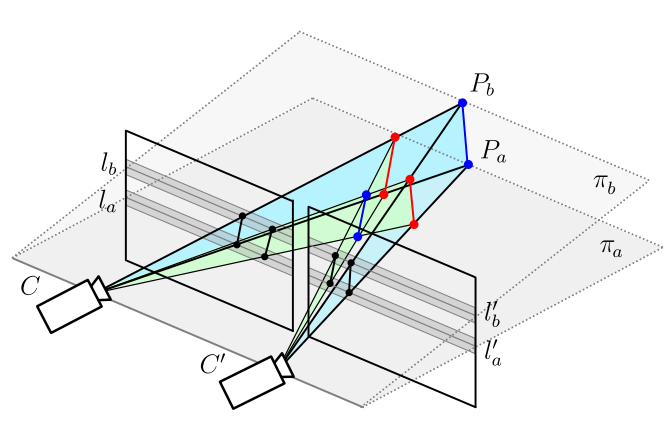

(a)

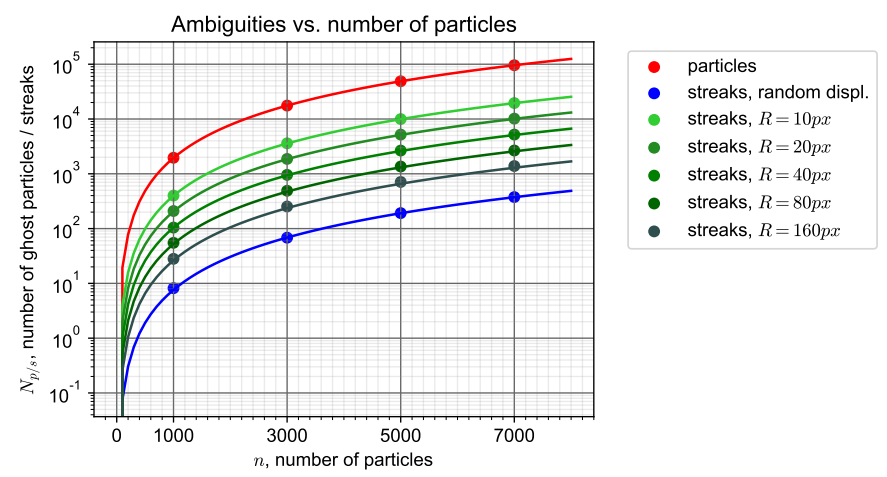

(b)

Figure 1: (a) Ghost streak generation in a two-camera setup and (b) number of reconstructed ghost particles and ghost streaks for different particle displacement scenarios, from estimates (lines) and simulations (dots)

within a given maximum allowable radius, within a distance of $[0, R]$. For the case of random displacements, the expected number of ghost streak generations is $N_{p}=2\left(n^{2}-n\right)(f / F)^{2}$, while for a displacement within $R$ we identify three mechanisms of ghost streak generation and derive the relevant probabilities.

3D reconstruction of the points and streaks is then performed using two methods: (a) the start and end points are reconstructed independently of each other, as in a time-resolved 3D-PTV setup, and (b) the endpoints are matched and reconstructed jointly. In the second case, while knowledge of the endpoint connectivity is maintained, the knowledge of the directionality of the streak is lost. Therefore, each of the $2 n$ points in one image can be matched to any of the $2 n$ points in the second image.

For these simulations, the maximum allowable tolerance for the epipolar constraint is set to $1 \mathrm{px}$, while 1 Mpx images are used, and we do not constrain the reconstruction volume. The tests are performed with 100 datasets containing 1'000 to 7'000 particles each. For the streaks, matches are accepted when both endpoints of a streak in one image can be matched to both endpoints of a streak in the second image. The resulting number of ghost streaks, compared to that of ghost particles, demonstrates the advantage of reconstructing line segments (streaks) instead of points (particles) and validates our probability analysis (Fig. 1(b)].

\section{Conclusions}

With long-exposure particle imaging in 3D-PSV, particle pathlines are obtained, instead of frozen particle positions as in conventional 3D-PTV and Tomo-PIV. We show that the knowledge of the endpoint connectivity in streak imaging presents advantages in ghost particle elimination by reducing the requirements for the number of cameras, particle tracking and high-speed aqcuisition. Streak reconstruction already results in lower numbers of ghost reconstructions in a two-camera setup, while the number of ghost streaks depends on the distribution of streak lengths, seeding density, epipolar constraint/reprojection error tolerance and camera configuration.

\section{References}

Maas HG (1992) Complexity analysis for the establishment of image correspondences of dense spatial target fields. International Archives of Photogrammetry and Remote Sensing 29:102-107

Novara M, Schanz D, Geisler R, Gesemann S, Voss C, and Schröder A (2019) Multi-exposed recordings for 3D Lagrangian particle tracking with Multi-Pulse Shake-The-Box. Experiments in Fluids 60:1-19

Rosenstiel M and Grigat RR (2010) Segmentation and classification of streaks in a large-scale particle streak tracking system. Flow Measurement and Instrumentation 21:1-7

Schanz D, Gesemann S, and Schröder A (2016) Shake-The-Box: Lagrangian particle tracking at high particle image densities. Experiments in Fluids 57:1-27 